\title{
Crystal structure of erbium vanadium tantal boride, $\operatorname{Er}\left(\mathrm{V}_{0.77} \mathrm{Ta}_{0.23}\right) \mathrm{VB}_{6}$
}

\author{
Yu. B. Kuz'ma*,I, Yu. Prots ${ }^{I I}$ and Yu. Grin ${ }^{I I}$ \\ 1 National University of L'viv, Analytical Chemistry Institute, Kyrylo and Mefodii Str. 6, 79005 L'viv, Ukraine \\ II Max-Planck-Institut für Chemische Physik fester Stoffe, Nöthnitzer Str. 40, D-01187 Dresden, Germany
}

Received February 24, 2003, accepted and available on-line April 23, 2003; CSD-No. 409691

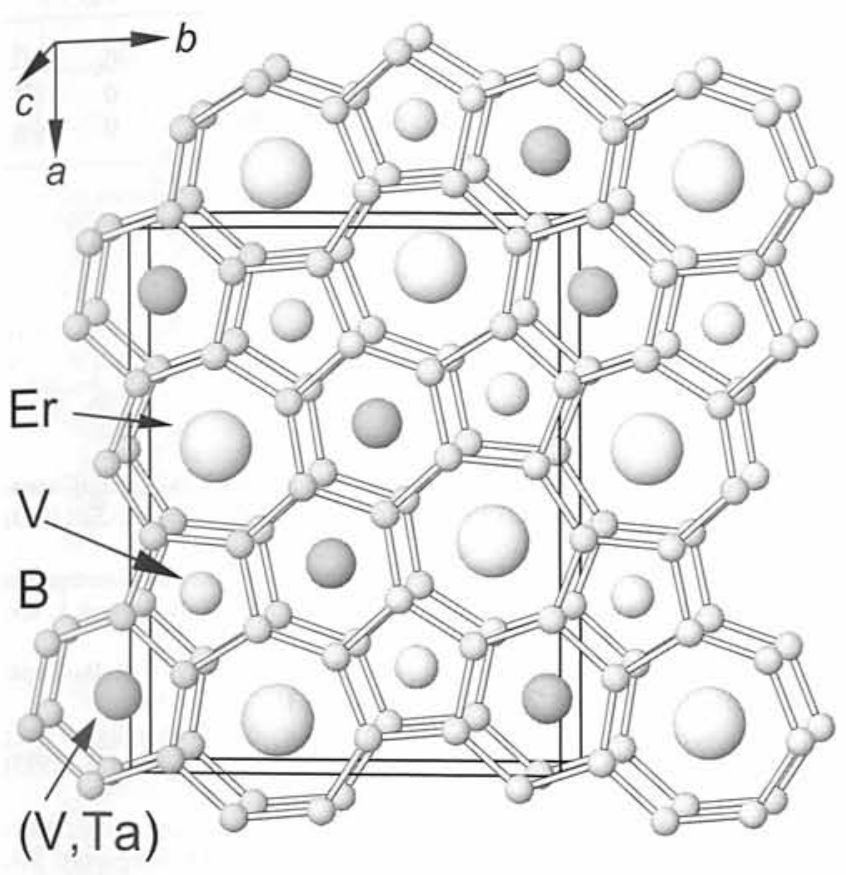

Abstract

$\mathrm{B}_{6} \mathrm{ErTa}_{0.23} \mathrm{~V}_{1.77}$, orthorhombic, Pbam (No. 55), $a=11.280$ (2) $\AA$, $b=8.940(2) \AA, c=3.390(1) \AA, V=341.9 \AA^{3}, Z=4$, $R_{\mathrm{gt}}(F)=0.034, w R_{\mathrm{obs}}(F)=0.034, T=293 \mathrm{~K}$.

\section{Source of material}

The title compound was obtained as by-product during systematic investigation in ternary Er-V-B system. A pellet pressed from erbium filings $(99.9 \%)$, vanadium powder $(99.5 \%)$, and crystalline boron ( $99.99 \%$ ) in ratio of 2:1:6 was wrapped in tantalum foil, sealed in quartz ampoule and sintered at $1270 \mathrm{~K}$. Needleshaped crystal were formed on contact surfaces of the pellet and tantalum foil. The presence of tantalum in the crystal was suspected during the structure refinement and was confirmed by EDAX. A detected atomic ratio between vanadium and sum of erbium with tantalum of $1.27(2): 1$ agrees with values of $1.44(1): 1$ obtained from crystal structure refinement.

\section{Experimental details}

The lattice parameters of $\operatorname{Er}\left(\mathrm{V}_{0.77} \mathrm{Ta}_{0.23}\right) \mathrm{VB}_{6}$ were determined from least-square procedure of 1978 reflections measured during the data collection.

\section{Discussion}

The crystal structure of $\operatorname{Er}\left(\mathrm{V}_{0.77} \mathrm{Ta}_{0.23}\right) \mathrm{VB}_{6}$ adopts $\mathrm{Y}_{2} \mathrm{ReB} 6$ type [1]. The boron atoms form two-dimensional planar nets of condensed five-, six-, and seven-membered rings. All prismatically coordinated centers between two slabs are filled by metals, according to the atomic radii, the largest $\mathrm{Er}$ and the smallest $\mathrm{V}$ atoms occupy the centers of heptagonal and pentagonal prisms, respectively. The hexagonal prisms contain statistically distributed $\mathrm{Ta}$ (intermediate size) and $\mathrm{V}$ atoms. Coordination polyhedra around $\mathrm{Er},(\mathrm{Ta}, \mathrm{V})$, and $\mathrm{V}$ atoms have 23,20 , and 17 vertices, respectively. Each boron atom is trigonal prismatically coordinated by metal atoms. The environment is completed by three additional boron neighbors, which are situated in the same plane as the central atom. The B-B bond lengths vary between 1.74(2) $\AA$ and 1.85(2) $\AA$ and agree very well with average distance of $1.776 \AA$ in $\alpha$-rhombohedral boron [2]. The shortest contacts between another pairs of atoms are $d(\mathrm{Er}-\mathrm{Ta}, \mathrm{V})=3.391(2) \AA, d(\mathrm{Er}-\mathrm{V})=$ $3.046(3) \AA, d(\mathrm{Er}-\mathrm{B})=2.631(1) \AA, d(\mathrm{Ta}, \mathrm{V}-\mathrm{V})=2.764(3) \AA$, $d(\mathrm{Ta}, \mathrm{V}-\mathrm{B})=2.426(1) \AA$, and $d(\mathrm{~V}-\mathrm{B})=2.254(9) \AA$. Ternary compounds with $\mathrm{Y}_{2} \mathrm{ReB}_{6}$ structure type are observed in numerous rare-earth transition metal boron systems: $\mathrm{RE}_{2} \mathrm{MB}_{6}(\mathrm{RE}=\mathrm{Y}$, $\mathrm{Gd}-\mathrm{Tm} ; \mathrm{M}=\mathrm{Ru}, \mathrm{Re}, \mathrm{Os}), \mathrm{RE}_{2} \mathrm{MB}_{6}(\mathrm{RE}=\mathrm{Yb}, \mathrm{Lu} ; \mathrm{M}=\mathrm{Ru}, \mathrm{Os}$ ), $\mathrm{Lu}_{2} \mathrm{FeB}_{6}, \mathrm{Yb}_{2} \mathrm{AlB}_{6}, \mathrm{U}_{2} \mathrm{MB}_{6}$ (M = Mo, W, Re, Os) [3]; $\mathrm{Sc}_{2} \mathrm{ReB}_{6}$ [4]; $\mathrm{RE}_{2} \mathrm{MoB}_{6}(\mathrm{RE}=\mathrm{Er}, \mathrm{Tm}, \mathrm{Yb}, \mathrm{Lu})$ [4,5]; $\mathrm{Lu}_{1.34} \mathrm{~V}_{1.66} \mathrm{~B}_{6}$ [6]; $\mathrm{Lu}_{2} \mathrm{WB}_{6}$ [7]. Nevertheless, a formation of isotypic phases was not detected during systematic investigation of ternary Er-V-B [8] and $\mathrm{Ta}-\mathrm{V}-\mathrm{B}$ [9] systems. $\operatorname{Er}\left(\mathrm{V}_{0.77} \mathrm{Ta}_{0.23}\right) \mathrm{VB}_{6}$ is the first quasi-ternary representative of $\mathrm{Y}_{2} \mathrm{ReB}_{6}$ type and the third example of described structure, where transition metal atoms have hexagonal-prismatic environment. Recently reported borides $\mathrm{Zr}_{2} \mathrm{CrB}_{6}$ and $\mathrm{ZrCr}_{2} \mathrm{~B}_{6}$ contain in the centers of hexagonal prisms $\mathrm{Cr}$ and statistically distributed $\mathrm{Cr}$ and $\mathrm{Zr}$ atoms, respectively [10].

Table 1. Data collection and handling.

$\begin{array}{ll}\text { Crystal: } & \text { metallic needle, size } 0.02 \times 0.03 \times 0.08 \mathrm{~mm} \\ \text { Wavelength: } & \text { Mo } K_{\alpha} \text { radiation }(0.7107 \AA) \\ \mu: & 377.5 \mathrm{~cm}^{-1} \\ \text { Diffractometer, scan mode: } & \text { Rigaku Mercury CCD, } \varphi / \omega \\ 2 \theta_{\text {max }} & 64.06^{\circ} \\ N(h k l)_{\text {measured }}, N(h k l)_{\text {unique: }}: & 2542,565 \\ \text { Criterion for } F_{\text {obs, }}, N(h k l)_{\text {gt: }}: & F_{\text {obs }}>4 \sigma\left(F_{\text {obs }}\right), 545 \\ N(\text { param })_{\text {refined: }} & 38 \\ \text { Programs: } & \text { WinCSD [11], ATOMS [12] }\end{array}$

\footnotetext{
* Correspondence author (e-mail: ukuzma@ franko.lviv.ua)
} 
Table 2. Atomic coordinates and displacement parameters (in $\AA^{2}$ ).

\begin{tabular}{llllll}
\hline Atom & Site & $x$ & $y$ & $z$ & $U_{\text {iso }}$ \\
\hline $\mathrm{B}(1)$ & $4 g$ & $0.237(1)$ & $0.202(1)$ & 0 & $0.005(2)$ \\
$\mathrm{B}(2)$ & $4 g$ & $0.213(1)$ & $0.518(2)$ & 0 & $0.004(2)$ \\
$\mathrm{B}(3)$ & $4 g$ & $0.081(1)$ & $0.241(2)$ & 0 & $0.005(2)$ \\
$\mathrm{B}(4)$ & $4 g$ & $0.067(1)$ & $0.447(2)$ & 0 & $0.005(2)$ \\
$\mathrm{B}(5)$ & $4 g$ & $0.320(1)$ & $0.371(2)$ & 0 & $0.006(2)$ \\
$\mathrm{B}(6)$ & $4 g$ & $0.024(1)$ & $0.907(2)$ & 0 & $0.007(2)$ \\
\hline
\end{tabular}

Table 3. Atomic coordinates and displacement parameters (in $\AA^{2}$ ).

\begin{tabular}{|c|c|c|c|c|c|c|c|c|c|c|}
\hline Atom & Site & $x$ & $y$ & $z$ & $U_{11}$ & $U_{22}$ & $U_{33}$ & $U_{12}$ & $U_{13}$ & $U_{23}$ \\
\hline $\mathrm{Er}$ & $4 h$ & $0.91475(5)$ & $0.32152(6)$ & $1 / 2$ & $0.0040(3)$ & $0.0021(2)$ & $0.0037(3)$ & $-0.0005(2)$ & 0 & 0 \\
\hline $\mathrm{V} / \mathrm{Ta}(1)^{a}$ & $4 h$ & $0.37194(1)$ & $0.55391(1)$ & $1 / 2$ & $0.0050(5)$ & $0.0041(5)$ & $0.0047(5)$ & $-0.0012(4)$ & 0 & 0 \\
\hline $\mathrm{V}(2)$ & $4 h$ & $0.1840(2)$ & $0.3555(2)$ & $1 / 2$ & $0.0052(8)$ & $0.0030(8)$ & $0.0044(8)$ & $0.0022(7)$ & 0 & 0 \\
\hline
\end{tabular}

a: $\mathrm{V} / \mathrm{Ta}(1)=0.768(3) \mathrm{V}+0.232 \mathrm{Ta}$

Acknowledgment. The authors are thanking Ms. K. Schulze for EDAX analysis.

\section{References}

1. Kuz'ma, Yu. B.; Svarichevskaya, S. I.: Crystal structure of compound $\mathrm{Y}_{2} \mathrm{ReB}_{6}$ and their analogous. Sov. Phys. Crystallogr. 17 (1972) 569-571.

2. McCarty, L. V.; Kasper, J. S.; Horn, F. H.; Decker, B. F.; Newkirk, A. E.: A new modification of boron. J. Am. Chem. Soc. 80 (1958) 2592.

3. Kuz'ma, Yu. B.; Chaban, N.F.: Binary and ternary systems containing boron. Metallurgiya, Moscow, 1990, p. 317.

4. Mykhailenko, S. I.; Chaban, N. F.: Kuz'ma, Yu. B.: Interaction of rare earths with VI and VII transition metals and boron. Powder Metall. Met. Ceram. 37 (1998) 99-106.

5. Chaban, N. F.; Mykhailenko, S. I.; Kuz'ma, Yu. B.: New compounds with $\mathrm{Y}_{2} \mathrm{ReB}_{6}$ structure type. Powder Metall. Met. Ceram. 37 (1998) 635-637.

6. Chaban, N.F.; Mykhailenko, S. I.; Davydov, V.M.; Kuz'ma, Yu. B: Phase equilibria diagram of Lu-V-B system. Powder Metall. Met. Ceram. 41 (2002) 162-168.

7. Chaban, N. F.; Mykhailenko, S. I.; Kuz'ma, Yu. B.: X-Ray studies on phase equilibria in $\{\mathrm{Tm}, \mathrm{Lu}-\mathrm{W}-\mathrm{B}\}$ ternary systems at $1070 \mathrm{~K}$. Powder Metall. Met. Ceram. 39 (2000) 251-255.

8. Chaban, N. F.; Nechaj, O. P.: Isothermal section of the erbium-vanadium-boron system at 1070 K. Powder Metall. Met. Ceram. 32 (1993) 539-540.

9. Kuz'ma, Yu. B.; Marko, M. A.; Petrovskaya, M. V.: X-Ray investigation of the ternary systems $\mathrm{V}-\mathrm{Ta}-\mathrm{B}, \mathrm{Nb}-\mathrm{Ta}-\mathrm{B}$ and $\mathrm{V}-\mathrm{W}-\mathrm{B}$. Visnyk L'viv. Univ., Ser. Khim. 13 (1972) 3-8.

10. Hillebrecht, H.; Gebhardt, K.: Neue Varianten des $\mathrm{Y}_{2} \mathrm{ReB}_{6}$-Typs: $\mathrm{Zr}_{2} \mathrm{CrB}_{6}$ und $\mathrm{ZrCr}_{2} \mathrm{~B}_{6}$. Z. Kristallogr. Suppl. 18 (2001) 156

11. Akselrud, L. G.; Zavalii, P. Y.; Grin, Yu. N.; Pecharsky, V. K.; Baumgartner, B.; Wölfel, E.: Materials Science Forum 133-136 (1993) 335-340.

12. Dowty, E.: ATOMS 5.1, A complete program for displaying atomic structures. By Shape software, 521 Hidden Valley Road, Kingsport, TN, 37663, USA 2000. 